\title{
O PREPARO DE ENFERMEIROS QUE ATUAM EM GRUPOS NA ÁREA DE SAÚDE MENTAL E PSIQUIATRIAA
}

\author{
The preparation of teamwork nurses in the area of mental \\ health and psychiatry.

\section{La preparación de enfermeros que realizan trabajo en grupo en el area de salud mental y psiquiatría.}

\begin{abstract}
RESUMO
Introdução: o trabalho em grupo tem sido enfatizado na possibilidade de promover a reabilitação psicossocial das pessoas com transtornos psíquicos. Objetivo: identificar como ocorre a formação do enfermeiro em grupos na área de Psiquiatria e Saúde Mental. Métodos: estudo qualitativo do tipo descritivo exploratório. Foram 26 enfermeiros os participantes dessa pesquisa, todos da rede de serviços de psiquiatria e saúde mental. Os dados foram coletados por meio de entrevista individual, seguindo roteiro com questões norteadoras. Para análise dos dados utilizou-se a ordenação e classificação dos dados e análise final. Resultados e discussão: as análises indicaram que o enfermeiro se desenvolve para atuar em grupos de forma mais vivencial, alguns fazem cursos extracurriculares e outros, leituras específicas. Considerações finais: há a necessidade de maior investimento durante a formação do enfermeiro durante a graduação em relação à coordenação de grupos, o que não difere dos dados encontrados na literatura a esse respeito.
\end{abstract}

Palavras-chave: Saúde Mental. Enfermagem. Educação em Saúde.

\begin{abstract}
Introduction: Teamwork has been emphasized to enable the promotion of psychosocial rehabilitation of people with psychic disturbances. Objective: to identify how nurse team education happens in the Psychiatric and Mental Health areas. Methodology: qualitative study of descriptive-exploratory. 26 nurses were participants in this research, all of them from the psychiatry and mental health services chain. The data were collected by means of individual interviews, followed by an outline of orientating questions. For the data analysis, the ordination and classification of the data and the final analysis were applied. Results and discussion: the analyses indicated that nurses develop themselves for teamwork mostly in an experience-based way; some take extracurricular courses and do specific readings. Final considerations: there is a need for more investment during the nurses' training while they are undergraduates, in relation to team coordination, and this does not differ from the data found in the pertinent literature.
\end{abstract}

Keywords: Mental Health. Nursing. Education in Health

\section{Resumen}

Introducción: el trabajo en grupo ha sido enfatizado porque ofrece la posibilidad de promover la rehabilitación psicosocial de las personas con trastornos psíquicos. Objetivo: identificar cómo ocurre la formación del enfermero en grupos en el área de Psiquiatría y Salud Mental. Métodos: estudio cualitativo de tipo descriptivo y exploratorio. 26 enfermeros participaron en esa pesquisa, todos pertenecientes a la red de servicios de psiquiatría y salud mental. Los datos fueron recolectados a través de entrevistas individuales, siguiéndo un guión con preguntas guía. Para el análisis de los datos, se utilizó la ordenación, la clasificación de los datos y el análisis final. Resultados y discusión: el análisis indicó que el enfermero se desarrolla para actuar en grupos más de forma vivencial, algunos hacen cursos extracurriculares y otros lecturas específicas. Consideraciones finales: existe la necesidad de hacer mayores inversiones durante la formación del enfermero en lo que a la coordinación de grupos se refiere, lo que no difiere de los datos encontrados en la bibliografía a este respecto.

Palabras-clave: Salud Mental. Enfermería. Educación en Salud.

${ }^{1}$ Enfermeira Especialista e Mestre em Psiquiatria e Saúde Mental pela Escola de Enfermagem da Universidade de São Paulo da USP de Ribeirão Preto/ SP. Brasil. E-mail: luspadini@hotmail.com, ${ }^{2}$ Professor Doutor do Departamento de Enfermagem Psiquiatria e Ciências Humanas da Escola de Enfermagem de Ribeirão Preto - USP. Brasil. E-mail: consouza@eerp.usp.br 


\section{INTRODUCÃO}

A formação de profissionais de saúde tem sido uma preocupação do governo, na medida em que a implantação de novas propostas de atuação no setor saúde implica a formação de profissionais mais comprometidos para atender a necessidade de saúde da população. Há um movimento de mudança do modelo de assistência, centrado no hospital, para promoção de saúde, prevenção, diagnóstico precoce, recuperação e reabilitação.

0 Ministério da Saúde reconhece como fundamental para transformar a assistência a formação do profissional de saúde sob a ótica da ação integral, acesso universal, equidade, participação e controle social.

Neste sentido, as unidades de ensino na área de saúde têm retomado suas propostas curriculares e investido em rever os seus Projetos Políticos Pedagógicos.

As Diretrizes Curriculares Nacionais do Curso de Graduação em Enfermagem apontam o trabalho em grupo como importante para a formação do enfermeiro, quando referem, no artigo 14, "que a estrutura do curso de graduação em enfermagem deve assegurar estímulo às dinâmicas de trabalho em grupos, por favorecerem a discussão coletiva e relações interpessoais"; e ainda, no artigo 5, que "a formação do enfermeiro tem por objetivo dotar o profissional dos conhecimentos requeridos para o exercício das seguintes competências e habilidades específicas: comunicar-se, tomar decisões, intervir no processo de trabalho, trabalhar em equipe e enfrentar situações constantes de mudanças, reconhecer-se como coordenador do trabalho de equipe de enfermagem". ${ }^{1}$

Neste sentido, compreendemos que o trabalho em grupo é de fundamental relevância para a formação do enfermeiro e para sua atuação profissional.

Na área de Saúde Mental, o trabalho em grupo tem sido enfatizado na possibilidade de promover a reabilitação psicossocial das pessoas com transtornos psíquicos e de favorecer o encontro de usuários e familiares. Por meio dos diálogos nos grupos, os usuários são encorajados a manifestar suas necessidades. ${ }^{2}$

Os grupos têm a capacidade de recriar ambientes familiares e sociais, possibilitando o desenvolvimento de habilidades e criações, desse modo, é um instrumento terapêutico eficiente. ${ }^{3}$

Dependendo da forma com que é conduzido e organizado, o recurso grupal pode ser usado em diversas situações e para diversos fins, melhorando muito a qualidade de vida das pessoas nos seus relacionamentos, na família, no trabalho e na sociedade como um todo. ${ }^{4}$
A formação na graduação pode constituir uma oportunidade para o exercício de reflexão sobre os contextos de realidade. Os grupos podem ser um recurso que instrumentalizam essa aprendizagem, pois são espaços de ação que permeiam as interações, dimensionando o elo entre 0 teórico e o vivencial. As situações de grupo na academia promovem uma aproximação com a situação do trabalho do profissional de saúde mental, envolvendo a prática em instituições e em comunidades. ${ }^{5}$

Porém, o enfoque teórico da maioria dos cursos de enfermagem está nas manifestações psicopatológicas visando preparar o aluno a atuar de acordo com a prática da psiquiatria clínica. ${ }^{6}$

Atualmente a utilização de grupos na assistência ao ser humano está ampliando-se, o que torna o conhecimento desse instrumental imprescindível para um melhor desempenho do profissional. Desse modo, o enfermeiro deve procurar formação específica que lhe oferecerá base para atuação assertiva e eficiente. ${ }^{4}$

Para coordenar grupos, são inerentes alguns atributos, como: "gostar e acreditar em grupos, amar as verdades, coerência, ética, respeito, paciência, continência, função de pensar; comunicação, empatia, síntese e integração". 7

Para que o trabalho em grupo obtenha sucesso, será necessário que o coordenador goste do que realiza, para não resultar em um desgaste pessoal e na não eficácia do trabalho.

0 coordenador deverá saber conter sua ansiedade e sua angústia e tentar suprir as necessidades dos outros integrantes do grupo. 0 profissional que trabalha em grupo precisa ter capacidade de comunicação, escuta, reflexão, respeito às diferenças, flexibilidade às mudanças, entre outras.

Tendo em vista o apontado anteriormente, este estudo buscou identificar como ocorreu a formação dos enfermeiros da rede de Psiquiatria e Saúde Mental de um município do interior paulista, para atuação em grupos.

\section{METODOLOGIA}

Trata-se de um estudo de natureza qualitativa do tipo descritivo exploratório. 0 tema deste estudo, formação do enfermeiro em grupo na área de Psiquiatria e Saúde Mental, foi extraído da dissertação de Mestrado intitulada "A inserção do enfermeiro no contexto de Saúde Mental: o trabalho com grupos". Os dados foram obtidos por meio de entrevistas individuais somente após a permissão dos enfermeiros 
mediante as condições da Resolução n 196/96 aprovada pelo Conselho Nacional de Saúde sobre pesquisa envolvendo seres humanos, ${ }^{8}$ e após apreciação do Comitê de Ética em Pesquisa do Hospital das Clínicas da Faculdade de Medicina de Ribeirão Preto - Universidade de São Paulo e assinatura dos participantes no Termo de Consentimento Informado Livre e Esclarecido, bem como a autorização dos locais de estudo. Para a coleta de dados e realização das entrevistas, foi utilizado um roteiro previamente elaborado com as seguintes questões norteadoras: Qual o tipo de preparo você tem para a coordenação de grupos em saúde mental? Onde você se prepara para desenvolver essa atividade?

As entrevistas foram realizadas em um único encontro em sala reservada nas instituiç̧ões com data e horário preestabelecidos. Duraram em média 15 minutos, foram gravadas e posteriormente transcritas na íntegra pelo próprio pesquisador.

A entrevista é o procedimento utilizado em pesquisa de campo em que o pesquisador busca obter informações contidas nas falas dos atores sociais. Ela se insere como meio de coleta de dados de fatores correlatos que vivenciam uma realidade que está sendo investigada. ${ }^{9}$

Os participantes desta investigação foram 26 enfermeiros da rede de serviços de Psiquiatria e Saúde Mental do município de uma cidade do interior paulista, que se constituía de serviços abertos, semiabertos e de internação integral.

Após a coleta de dados e transcrição das entrevistas, o material foi organizado, lido quantas vezes foram necessárias, sendo realizada a identificação de temas, provenientes dos depoimentos. Após isso, eles foram analisados conforme os objetivos desta investigação.

A análise do material coletado na pesquisa qualitativa deve ser feita com três finalidades distintas: identificar e conhecer as informações; confirmar ou refutar os pressupostos da pesquisa e, em um terceiro momento, ampliar o conhecimento sobre o tema estudado. ${ }^{9}$

Classificar significa organizar ou ordenar em uma série de diferentes dados de classes o todo ou o universo estudado, dividido em partes, agrupando os dados em categorias para serem analisados. ${ }^{10}$

$\mathrm{Na}$ análise e discussão dos dados, embasamo-nos passos propostos por Minayo:

1) Ordenação dos dados: reunir todo 0 material, ou seja, organizar os dados (início da classificação);

2) Classificação dos dados: leituras exaustivas do conteúdo transcrito das entrevistas; apreender as ideias centrais e estabelecer as categorias empíricas do estudo;
3) Análise final: articular o material teórico e o empírico da pesquisa, orientada pelos objetivos da investigação.

Para a elaboração dos temas, foram feitas leituras exaustivas dos dados coletados e uma análise das temáticas que emergiram nas entrevistas, relacionadas à revisão da literatura e aos objetivos da pesquisa. No atual estudo, realizamos um recorte e enfocamos o tema "formação do enfermeiro em grupos na área de saúde mental e psiquiatria".

\section{RESULTADOS E DISCUSSÃO}

Do total de 44 enfermeiros admitidos nos serviços, 26 (59\%) aceitaram participar da pesquisa. Todos os entrevistados participam ou já participaram de atividades grupais em seu serviço. Dos sujeitos envolvidos, 15 (57,7\%) estão no quadro de profissionais das unidades de internação integral, 9 (31\%), nos serviços abertos e $2(7,7 \%)$, nos serviços semiabertos.

Dos 26 enfermeiros, 24 são do sexo feminino $(92,4 \%)$ e 2 são do sexo masculino (7,6\%). A idade deles variou de 24 a 52 anos. Analisando a instituição formadora, 15 (57,7\%) concluíram o curso de graduação em enfermagem em escola publica e 11 (42,3\%), em escolas privadas. Quanto ao ano de formação, variou de 1979 a 2003.

Com relação à atuação dos enfermeiros nos grupos, $6(23 \%)$ referiram ser coordenadores de grupo; $6(23 \%)$, coterapeutas; 2 (7,7\%), observadores e 12 (46,3\%) referiram ser participantes nos grupos realizados nos serviços de psiquiatria e saúde mental.

Foi possível constatar, por meio das respostas dos enfermeiros, que a formação em grupos é fundamental para o bom desempenho da realização da atividade grupal, que esta inclui o desenvolvimento nos passos teórico-técnico e pessoal, e que o preparo, ocorre mais na vivência do que por meio de uma formação mais específica.

Segundo os participantes, o curso de graduação em enfermagem, não dá subsídios para o exercício dessa atividade. Alguns enfermeiros buscam cursos específicos, outros fazem somente leitura sobre o assunto. Notam que não há investimento das instituições de saúde, de um modo geral, para a formação do profissional em grupos.

Nas entrevistas a seguir, pode-se observar que os enfermeiros pesquisados apontam a necessidade de ser mais enfatizada na graduação a questão sobre grupos:

...mas precisa ter formação, precisa ter pelo menos uma aproximação com alguns conhecimentos de 
grupo... acho que muito é da personalidade do enfermeiro de gostar de grupos... fiz especialização em Psiquiatria e Saúde Mental, Mestrado em Psiquiatria... acho que ainda na formação do enfermeiro trabalha-se muito pouco a questão de grupos...tem que ter essa formação... na graduação, porque tudo a gente faz em grupo(E10).

Eu acho que o enfermeiro tem uma base muito restrita a nivel de preparo com grupos, desde a formação em si... a nível de grupo, a gente procura ler, participei de disciplinas... gostaria de futuramente participar de algum curso para eu estar me preparando. A instituição não oferece nada... não oferece curso algum( $E 3)$.

...não tenho nenhum preparo, acho que tira muito ponto, acho que ficou falho na faculdade, esse negócio de grupos, como coordenar e nem achava que era tanto nossa função(E 21).

0 enfermeiro precisa demonstrar competência no manejo da atividade grupal porque, em algumas situações, 0 interesse da instituição é insignificante, colocando obstáculos à atividade. ${ }^{11}$

No país, são poucos os cursos de enfermagem que tratam do conteúdo "grupos" na graduação, embora, nas novas Diretrizes Curriculares, esteja regulamentada a necessidade de capacitação profissional para o trabalho com grupos. ${ }^{12}$

Está determinado na Resolução das Diretrizes Curriculares Nacionais do Curso de Graduação em Enfermagem de sete de novembro de 2001, artigo 14, parágrafo sete: "0 estímulo às dinâmicas de trabalho em grupos, por favorecerem discussão coletiva e as relaç̃̃es interpessoais '".

Em estudo realizado no ano de 2000, a autora menciona que, muito embora a enfermagem tenha como característica o relacionamento com pessoas, o enfermeiro poucas vezes faz uso do recurso grupal. Refere que o profissional não está instrumentalizado para essa função, pois a grade curricular das escolas de enfermagem pouco contempla o coordenar grupos, embora as aulas teóricas e os estágios ocorram em grupos. ${ }^{13}$

Em um outro estudo, as autoras destacam que o fato de estarem preparando enfermeiros há mais de uma década levou-as a questionar alguns aspectos da formação desse profissional. Vislumbram o aprofundamento de formas e estratégias para a formação de modo pleno e consciente, privilegiando o desenvolvimento técnico e pessoal.
Mencionam, ainda, que as tendências atuais exigem do profissional o perfil de uma pessoa capaz de investir no seu autoconhecimento e que a estrutura da disciplina "Saúde Mental" pode viabilizar esse processo, funcionando como elemento facilitador. ${ }^{14}$

A proposta de ensino pautada na metodologia de grupos operativos mostrou-se eficiente para poder constituir-se em articuladora da ação docente e profissional da enfermagem em formação. ${ }^{15}$

Nos depoimentos seguintes, os enfermeiros apontam para um conhecimento adquirido com a vivência, por observação e realização de algumas leituras sobre 0 assunto:

...na prática, com a experiência dos outros, supervisão, mas não fiz nenhum curso, apesar de ter a intenção de fazer... então meu aprendizado foi mais na prática(E7).

...estou indo mais nas tentativas e erros... tem a supervisão com o pessoal que já tem muita experiência de grupo(E 11).

Eu via como as outras pessoas faziam... foi um conhecimento da prática, se estava certo ou errado eu não sei(E 13).

...aprendendo mais na observação... por não ter tido formação...(E 16).

Apesar de o enfermeiro utilizar o recurso grupal em sua prática, muitas vezes, faz isso usando somente a intuição, não buscando recursos teóricos e práticos para uma ação mais eficiente. ${ }^{16}$

0 profissional que parte do empírico, buscando acertar, pode ter dificuldades em exercer o papel de coordenador, ainda que a vivência contribua para tal, o mesmo estaria resguardado se tivesse algum conhecimento da dinâmica humana e dos grupos. ${ }^{11}$

Alguns enfermeiros, questionados sobre a formação em grupos em saúde mental, mencionam a busca de cursos específicos sobre grupos, supervisões, especialização em Enfermagem Psiquiátrica e Saúde Mental, leituras específicas, o desenvolvimento do autoconhecimento, a importância do gostar de grupos, a participação em outros grupos e supervisões como treino para atuar na atividade grupal:

Eu fiz curso de Pichon há uns anos atrás, mas foio treinamento aqui... foi de assistir grupos...(E 5). 
... a minha formação me deu esse preparo, tenho especialização em Enfermagem Psiquiátrica e Saúde Mental, Mestrado e Doutorado em Enfermagem Psiquiátrica... fiz formação em grupos operativos... e fiz estudo de grupos com um profissional da área... além disso, fiz psicodrama e formação de expressão corporal... fizemos muitas discussões sobre o desenvolvimento do ser humano em atividades grupais. Isso facilitou para que eu pudesse estar habilitado aos vários e diferentes grupos no qual eu participo e tenho que coordenar, isso tudo deu-me embasamento para eu fazer trabalhos de grupos(E6).

...supervisões... temos um referencial teórico que é o Yalom, já fiz cursos de curta duração sobre esse referencial e de como estar aplicando psicoterapia no grupo... na graduação, tive alguns referenciais teóricos e dinâmica...(E 14).

...fiz vários cursos de grupo mais voltados para 0 psicodrama (E15).

...comecei a fazer um curso introdutório em grupos operativos segundo Pichon Riviéri... o que eu procuro fazer éler muito... leio muito Bion, que para mim é um dos autores mais importantes quando se fala de grupos... fiz especialização, participei de grupos... aprendi muito com isso...(E 4).

Em um estudo bibliográfico sobre a participação de enfermeiros como coordenadores de grupos, foi destacada pelas autoras a necessidade de o enfermeiro buscar o preparo adequado para o bom desempenho de coordenação de grupos.

Acreditam que esse preparo começa com 0 autoconhecimento e com conhecimentos específicos sobre dinâmica de grupos. ${ }^{17}$

Em um outro trabalho realizado por pesquisadora enfermeira, sobre o processo grupal em enfermagem e as possibilidades e limites, a autora aponta para a real importância de buscar embasamento para o desenvolvimento da atividade grupal, no sentido de prover de forma adequada e efetiva a realização da atividade grupal. ${ }^{11}$

Neste sentido, fica evidente que o enfermeiro precisa buscar conhecimentos que vão além da graduação, que lhe deem respaldo para trabalhar com grupos em psiquiatria e saúde mental. Os participantes do atual estudo enfatizam a necessidade da formação em grupos como fundamental para o bom desempenho da atividade grupal, constatando-se uma necessidade da melhora dos cursos de graduação no provimento desse recurso.

\section{CONSIDERAÇÕES FINAIS}

Os resultados deste estudo possibilitaram constatarmos que a formação do enfermeiro em grupos é fundamental para o bom desempenho da atividade grupal, embora a literatura aponte que a graduação em enfermagem não dá subsídios ao aluno para a coordenação de grupos. Atualmente, os enfermeiros que buscaram esse preparo fizeram cursos e/ou estágios em grupos de outros serviços. Outros ainda acreditam mais na experiência empírica para 0 desenvolvimento da atividade. Em algumas situações, não há investimento da instituição de saúde no profissional, não colaborando para sua qualificação.

Os enfermeiros pesquisados que referiram estar preparados para a utilização do recurso grupal citaram que fizeram cursos extracurriculares e estágios em outros grupos e que a supervisão é uma ferramenta no aprimoramento da realização grupal.

A nosso ver, o enfermeiro que atua em Psiquiatria e Saúde Mental deve preparar-se para atuar em grupos, uma vez que essa atividade é muito utilizada nessa área; também deve haver mais investimento das instituições de saúde e ensino voltado para a realização de grupos na assistência.

Os resultados apontam para a necessidade de um melhor preparo dos enfermeiros para o trabalho com grupos, principalmente em relação à sua coordenação, o que não difere dos dados encontrados na literatura a esse respeito.

Pensamos que a pesquisa realizada poderá contribuir para que os profissionais das instituições de saúde e ensino reflitam sobre as questões da formação do enfermeiro na coordenação de grupos em saúde mental para a melhoria da qualidade da assistência.

A formação do enfermeiro para atuação em grupos na área de Psiquiatria e Saúde Mental precisa ser repensada, pois, segundo alguns participantes do estudo, para se especializarem, precisam buscar cursos, e isso demanda tempo, tempo esse que nem sempre é possível devido às demandas do serviço e à política da instituição.

A temática grupo carece de um melhor enfoque tanto na assistência como no ensino, para que haja uma aplicação apropriada desse recurso como fonte das ações de enfermagem. 


\section{REFERÊNCIAS}

1. Ministério de Educação e Cultura (BR). Diretrizes Curriculares Nacionais do Curso de Graduação em Enfermagem. Conselho Nacional de Educação. Câmara de Educação Superior. Resolução CNE/CES 3/ 2001. Diário Oficial da República Federativa do Brasil. [on-line] 2001; [citado 10 dez 2006]; [aprox. 03 telas]. Disponível em :http:// www.portal.mec.gov.br/cne/arquivos/pdf/CES03.pdf

2. Kantorski LP, Machado AT, Oliveira CA. Centro de atenção psicossocial- reinventando o cuidado em saúde mental. Texto \& Contexto Enferm 2000; 9(1): 233-43.

3. Maximino VSA. A constituição de grupos de atividades com pacientes graves. Rev Terap Ocup 1995; 1(1): 27-32.

4. Godoy MTH. Análise da produção científica sobre a utilização de atividades grupais no trabalho do enfermeiro no Brasil: 1980 a 2003. [dissertação de mestrado]. Goiânia (GO): Faculdade de Enfermagem/ UFG; 2004.

5.Japur M, Loureiro SR. Formação acadêmica na área de saúde mental: o dispositivo grupal. In: Marturano EM, Loureiro SR, Zuardi AW. Estudos em saúde mental. Ribeirão Preto (SP): FMRPUSP; 1997. p. 299-317.

6. Souza MCBM. Enfermeiros assistências das instituições psiquiátricas de Ribeirão Preto: caracterização, formação e atuação [tese de doutorado]. Ribeirão Preto(SP): Escola de Enfermagem/USP; 1999.

7. Zimerman DE, Osorio LC, organizadores. Como trabalhamos com grupos. Porto Alegre (RS): Artes Médicas; 1997.

8. Palácios M. Ética na pesquisa em seres humanos. In: Palácios M, Martins A, Pegorano OA. Ética, ciência e saúde: desafios da bioética. Petrópolis(RJ): Vozes; 2001. p. 164-75.
9. Minayo MCS. 0 desafio do conhecimento: pesquisa qualitativa em saúde. São Paulo (SP): Hucitec /Abrasco; 1996.

10. Marconi MA, Lakatos EM. Técnica de pesquisa. São Paulo (SP): Atlas; 2002.

11. Munari DB. Processo grupal em enfermagem: possibilidades e limites. Rev Bras Enferm 1997; 50(1): 37-52.

12. Rocha BS, Munari DB. 0 ensino da dinâmica de grupo nos cursos de graduação em enfermagem no Brasil: nota prévia. Anais do $7^{\circ}$ Encontro de Pesquisadores em Saúde Mental. Encontro de Pesquisadores em Saúde Mental. $6^{\circ}$ Encontro de Especialistas em Enfermagem Psiquiátrica; 2002 mar 25-28; Ribeirão Preto(SP), Brasil. Ribeirão Preto(SP): 2002. p. 184.

13. Lucchese R. Grupo operativo como estratégia pedagógica em um curso de graduação em enfermagem: um espaço continente das vivências dos alunos quartanistas. [dissertação de mestrado]. São Paulo (SP): Escola de Enfermagem / USP; 2000.

14. Esperidião E, Munari DB, Stacciarini JMR. Desenvolvendo pessoas: estratégias facilitadoras para o autoconhecimento na formação do enfermeiro. Rev Latino-am Enfermagem [on-line] 2002 jul; [citado 20 dez 2006] 10(4): [aprox. 14 telas]. Disponível em: http://www.scielo.br

15. Ciampone MHT. Grupo operativo: construindo as bases para o ensino e a prática na enfermagem [tese de livre docência]. São Paulo (SP): Escola de Enfermagem/ USP; 1998.

16. Munari DB, Rodrigues ARF. Enfermagem e grupos. Goiânia (GO): $\mathrm{Ed} A B ; 1997$.

17. Spadini LS, Souza MCM. Grupos realizados por enfermeiros na área de saúde mental. Esc Anna Nery Rev Enferm 2006; 10(1): 132-38.

\section{NOTA}

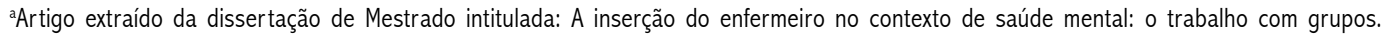

